\title{
Childhood interstitial lung disease: A diagnostic challenge for the pediatrician
}

\author{
Laura Larisa Dracea ${ }^{1,2}$ \\ ${ }^{1}$ Clinical Pediatric Department II, Clinical Emergency Children's Hospital, Brasov, Romania \\ ${ }^{2}$ Medical and Surgical Specialties Department, Medicine Faculty, \\ Transilvania University, Brasov, Romania
}

\begin{abstract}
Childhood interstitial lung disease (chILD) is represented by a very heterogenous group of rare genetic and lung development disorders that are distinct from those described in adult pulmonology $(1,2)$. Even complete understanding is still limited, advances in classification have been recently made (3).

The term of ILD (interstitial lung disease) is nonspecific and describes features of remodeling of the lung interstitium and distal airspaces. A systematic approach to diagnosis is needed, that includes careful history and physical examination, exclusion of common conditions presenting with similar features and early referral to expert centers (1). Timely diagnosis is important for patient management and prognosis, even effective and standardized interventions are often lacking.

One of the more recent described disorders, neuroendocrine cell hyperplasia of infancy (NEHI), will be presented with emphasis on clinical recognition.
\end{abstract}

Keywords: interstitial lung disease, children, prognosis, neuroendocrine cell dysplasia

\section{INTRODUCTION}

ChILD is represented by a very heterogenous group of rare genetic and lung development disorders, associated with significant morbidity and mortality. Even important advances were made in understanding the underlying pathological processes in infants and children, considerably different from those met in adult ILD, the understanding remains limited. Disorders that appear early in life may be distinct from those occurring later in childhood, even there are some overlaps. Nonspecific respiratory symptoms, diffuse pulmonary infiltrates, make exclusion of common conditions presenting in similar ways, an essential preliminary step in diagnosis $(1,2)$. The term of ILD (interstitial lung disease) is nonspecific and describes features of remodeling of the lung interstitium and distal airspaces. Some entities, characterized by primary pathology outside of the interstitium (vessels, epithelium), are referred to as diffuse lung disease (DLD) (3).
The underlying processes are growth developmental issues and immune dysregulation or compromise (4).

New approaches to classification, diagnosis and management include several steps: recognition of different natural history compared to adults (a), recognition of unique phenotypes, especially in infants (b), discovery of genetic causes of pediatric ILD (c), and advances in diagnostic approaches (d) (3).

Clinical signs as tachypnea, occurring in 75$93 \%$ of patients $(5,6)$, hypoxemia, crackles and cough (6) are the main features in DLD, but some of children may have wheezing and normal breath sounds. Failure to thrive may be a common feature for infants (5).

The term of "chILD syndrome" has been adopted in order to prompt investigation of common phenotypes characterized by at least three of the following four criteria: (a) respiratory symptoms (e.g., rapid/difficult breathing, cough, exercise in- 
tolerance); (b) respiratory signs (e.g., resting tachypnea, retractions, crackles, digital clubbing, failure to thrive, respiratory failure); (c) hypoxemia, and (d) diffuse anomalies on the chest x-ray or chest computed tomography (CT) scan.

\section{CASE REPORT}

Our patient, full term male, with normal growth and development, hyperactive, first presented at the age of 3 years and 6 months with a history of intermittent tachypnea and hypoxemia correlated with RTIs, sometimes accompanied by wheezing. Some more severe episodes, needed hospitalization during infancy. Acute episodes were accompanied by respiratory distress and had incomplete improvement after treatment with antibiotics and systemic corticosteroids. The child had normal growth, was hyperactive and persisted in having episodes of tachypnea and hypoxemia during sleep.

The past medical history, at the age of two years, in attempt to establish a cause of persistent tachypnea and recurrent nocturnal episodes of desaturation, led to suspicion of intrabronchial foreign body, but bronchoscopy, was negative. Cardiologic evaluation was normal. Viral induced wheezing was suspected and Flixotide $125 \mathrm{mcg}$ b.i.d. and Singulair was recommended, but did not influence the outcome.

At current presentation, during a mild LRTI, he experienced periods of tachypnea during the day, with desaturation between $83 \%$ and $91 \%$ more accentuated during sleep, unfrequent cough and discrete wheezing that improved with supplemental oxygen therapy. Normal inflammatory markers, normal WBC determined minimal treatment, with no antibiotics. There was no improvement with systemic corticosteroids..
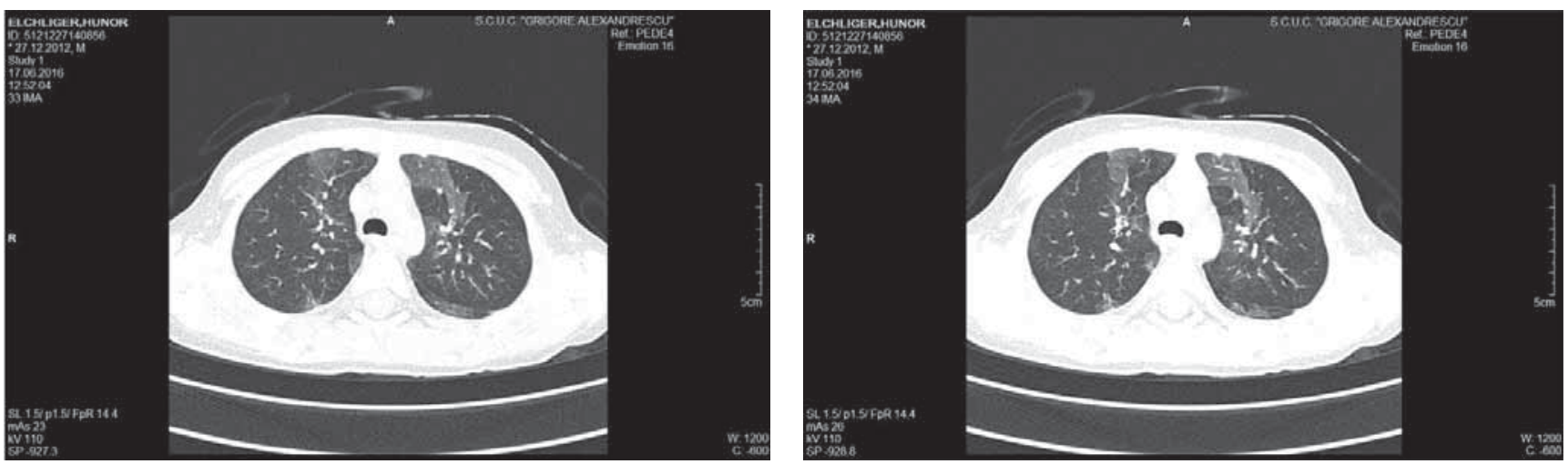

FIGURE 1. Air-trapping of left lower lobe and right lower lobe. Inhomogeneous appearance of upper left lobe, right upper lobe and middle lobe, ground glass hyperdense non-systematized opacities. Bronchiectasis of the medial segment of the middle lobe and lingula.
Exclusion of other chronic respiratory diseases was mandatory. Gastroesophageal reflux and allergy work-up were negative (no sensitization to inhaled allergens, normal total Ig E), and no immune deficit was noted. Normal sweat test excluded cystic fibrosis. Chest X-ray showed perihilar interstitial markings and hyperinflation in the lower fields of the lungs. Polysomnography showed nocturnal desaturation and alternation of short cycles of hypoventilation/hyperventilation. Cerebral MRI was normal.

Persistent tachypnea with desaturation with onset during infancy, triggered by RTIs, led to high suspicion for chILD, which prompted for a chest CT (Fig. 1).

According to the specific high resolution CT (HRCT) findings and clinical symptoms, diagnosis of "NEHI syndrome" was established, with support of expert oppinion of a radiologist and pediatric pulmonologist.

After spontaneous improvement of the current illness, the patient was discharged with supplemental home oxygen therapy.

In the following period, clinical status improved consistently, with long periods with no need of supplemental oxygen. A recent exacerbation, at the age of 6 years, triggered by a viral respiratory infection, determined hospital admission. Oxygen saturation decreased mildly during the day, and more, during the night. Mild wheezing and unfrequent cough, some crackles at auscultation of the chest were observed at presentation. Interstitial markings and discrete bronchiectasis of the middle lobe and lingula, hyperinflation of the bases of lungs were noted on the chest X-ray. Slightly positive inflammatory markers have been noticed.

This episode prompted for supplemental oxygen therapy during hospitalization, a short course of 
systemic corticosteroids that led to clinical improvement and discharge with nocturnal need of supplemental oxygen for only a few days. A repeated chest CT-scan and lung function measuring were recommended.

\section{DISSCUSION}

First described by Robin Deterding in 2005 (7), NEHI, known also as "persistent tachypnea of infancy", often remains undiagnosed because of phenotypical variability and mild symptoms, unless masquerading recurrent respiratory infections encountered during infancy.

NEHI is a rare form of ILD which involves small airways where clinical suspicion based on tachypnea and hypoxemia is an important clue for diagnosis. It may begin during infancy with persistent cough, tachypnea and hypoxemia after a respiratory infection and tend to slowly improve over time.

Diagnosis is based on clinical, evaluation, imaging and lung biopsy. As stated in recent literature, HRCT appears to be the most reliable non-invasive imaging that can suggest the diagnosis $(8,9)$, and that could potentially obviate the need for lung biopsy. The findings of multi-lobar ground-glass opacity predominantly involving the right middle lobe and lingula, as well a mosaic pattern of airtrapping are characteristic (83\% sensitivity, $100 \%$ specificity of radiolgy reports being encountered), thus differentiating between other types of ILD (10).

In some cases, as NEHI, CT findings are highly suggestive or even characteristic and may also provide prognostic information (e.g., NEHI has a favorable prognosis, whereas growth abnormalities and surfactant mutations carry substantial mortality).

Diagnosis on biopsy is based on essentialy normal histology on standard staining with increase in Bombesin-positive cells on specific staining. However, this only pathologic consistent finding may be seen in a variety of disorders as bronchopulmonary dysplasia, bronchiolitis and pulmonary hemosiderosis that have specific features which would preclude diagnostic confusions.
Familial cases of NEHI suggested a genetic origin, but, to date, no specific mutation has been identified yet, even an association with a mutation in the NK2 homeobox 1 (NKX2.1/TTF-1) involved in lung development, surfactant homeostasis and innate immune responses was discovered (11).

Long term outcome is usually favorable, with most patients improving gradually, as seen in the presented case. Sometimes persistent airway obstruction mimicking asthma and relapses triggered by respiratory infections have been reported.

\section{CONCLUSIONS}

Childhood ILD, a large spectrum of rare and hetereogenous disorders, are widely under-recognised and differ substantially from adult ILD.

A systematic, stepwise approach to patients whith suspected chILD, is crucial in establishing the diagnosis. The clinical context may ease the differential diagnosis, when starting from history and careful physical examination of those children that meet mentioned criteria, in order to be included in the category of chILD syndrome. Further investigation, as genetic testing, CT, bronchoscopy and bronchoalvelar lavage, lung function tests in older children and lung biopsy, may warrant a diagnosis.

Present collaborative efforts in the field of research and clinical care are made in order to provide insights in the molecular basis of chILD and identify targets for therapeutic interventions.

\section{ACKNOWLEDGEMENTS}

Prof. Andrew R. Colin, Director of the Division of Pediatric Pulmonology, Miller School of Medicine, University of Miami, USA

Prof. Dr. Robert P. Guillerman, Radiology Department, Texas Children's Hospital, Baylor College of Medicine, Houston, USA

Colaborators from the Clinical Emergency Children's Hospital Grigore Alexandrescu Bucharest: Simona Mosescu, MD, PhD (Pediatric Department), Tania Butufei, MD (Radiology Department), and Medicover Center Bucharest: Mihaela Oros, MD.

\section{Conflict of interest: none declared} Financial support: none declared

\section{REFERENCES}

1. Spagnolo $P, B$ Bush $A$. Interstitial lung disease in children younger than 2 years. Pediatrics 2016; 137(6)

2. Griese M. Chronic interstitial lung disease in children. Eur Respir Rev 2018; $27: 170100$

3. Kurland G, Deterding RR, Hagood JS et al. An Official American Thoracic Society Clinical Practice Guideline: Classification, Evaluation, and Management of Childhood Interstitial Lung Disease in Infancy. Am J Respir Crit Care Med 2013.188: 376-394 
4. Bush A, Nicholson AG. Pediatric Interstitial lung disease. Eur Respir Mon 2009, 46, 319-354.

5. Deutsch GH, Young LR, Deterding RR et al. Diffuse lung disease in young children: application of a novel classification scheme. Am J Respir Crit Care Med 2007; 176: 1120-1128.

6. Clement A. ERS Task Force. Task Force on Chronic Interstitial Lung Disease in Immunocompetent Children. Eur Respir J 2004; 24:686697.

7. Deterding RR, Pye C, Fan LL, Langston C. Persistent tachypnea of infancy is associated withneuroendocrine cell hyperplasia. Pediatr Pulmonol. 2005;40(2):157-165

8. Guillerman RP. Imaging of Childhood Interstitial Lung Disease. Pediatr Allergy Immunol Pulmonol 2010. 23(1) :43-6
9. Lee J, Sanchez TR, Zhang Y et al. The role of high-resolution chest $\mathrm{CT}$ in the diagnosis of neuroendocrine cell hyperplasia of infancy: a rare form of pediatric interstitial lung disease. Resp Med Case Rep 2015; 16.101-103

10. Brody AS, Guillerman RP et al. Neuroendocrine Cell Hyperplasia of Infancy: Diagnosis with High-Resolution CT. AJR AM J Roentgenol 2010; 194(1): 238-244

11. Young LR, Deutsch GH, Bokulic RE et al. A mutation in TTF1/NKX2.1 is associated with familial neuroendocrine cell hyperplasia of infancy. Chest. 2013;144(4):1199-1206 